\title{
DERIVACIÓN URINARIA EXTRA-ANATÓMICA MEDIANTE CATÉTER SUBCUTÁNEO
}

\author{
P. PÉREZ SANZ, B. POZO MENGUAL, R. MARCÉN LETOSA, J. PASCUAL SANTOS, \\ F.J. BURGOS REVILLA
}

Servicio de Urología y Nefrología. Departamento de Ciencias Morfológicas. Universidad Alcalá de Henares. Hospital Ramón y Cajal. Madrid.

Actas Urol Esp. 28 (4): 314-317, 2004

\section{RESUMEN}

DERIVACIÓN URINARIA EXTRA-ANATÓMICA MEDIANTE CATÉTER SUBCUTÁNEO

La derivación urinaria mediante nefrostomía percutánea ha sido uno de los métodos de tratamiento paliativo más empleados en el paciente con insuficiencia renal crónica secundaria a uropatía obstructiva de origen neoplásico.

Factores psicológicos y sociales hacen que esta medida sea rechazada por algunos pacientes incluso en situaciones en las cuales es la única medida que prolongaría la supervivencia. El empleo de stent ureterales beneficia a un porcentaje de pacientes, no siendo posible su empleo en todas las ocasiones.

La derivación con catéter subcutáneo es una alternativa sencilla y bien tolerada. El siguiente caso clínico describe la implantación de un catéter de derivación subcutánea en un varón de 60 años, trasplantado renal, tras fracaso de manejo de estenosis ureteral severa mediante endoscopia y reimplantación.

PALABRAS CLAVE: Trasplante renal. Derivación urinaria. Estenosis ureteral.

\section{ABSTRACT \\ EXTRA-ANATOMIC URINARY DIVERSION BY SUBCUTANEOUS CATHETER}

Percutaneous nephrostomy has been one of most used palliative method of urinary diversion to treat cronic renal failure in neoplasic patients.

Psychological and social factors meke this measure to be rejected by some patients even sin situations where this is the only action that would make their survival longer. The utilization of ureteral stents provides benefits to a certain percentage of patients, not being possible its usage in all the occassions.

Urinary subcutaneous diversion can be a simple and well tolerated alternative for the patient. The following clinic case describes and approach implementing a subcutaneous stent in a 60-yold male with a severe ureteric obstruction after failed management by endoscopy or open surgery.

KEY WORDS: Renal transplantation. Urinary diversion. Uretheral stenosis. 
$\mathrm{L}$ a derivación urinaria mediante nefrostomía permanente ha sido uno de los métodos de tratamiento paliativo más empleados en el paciente con insuficiencia renal crónica secundaria a hidronefrosis por uropatía neoplásica. Aunque en ocasiones es el único método que prolongaría la supervivencia, su empleo es rechazado por los pacientes por diversos factores sociales y psicológicos, al requerir una bolsa externa recolectora, con difícil manejo en su domicilio.

El drenaje mediante nefrostomía así mismo tiene una serie de complicaciones como la hematuria intermitente, dolor, infección de orina, existiendo la posibilidad de desplazamiento o salida accidental de la misma, requiriendo su recolocación y/o sustitución.

Mediante la colocación de stent ureterales convencionales, se ha aportado una mejor calidad de vida a este grupo de pacientes, sin embargo su empleo está limitado por la necesidad de cateterización del uréter previa a la colocación del stent.

La derivación nefrovesical extra-anatómica mediante catéter subcutáneo es otra alternativa sencilla y bien tolerada. Aunque su mayor indicación es como método de resolución de la uropatía obstructiva de origen neoplásico ${ }^{3,9,10}$ (cáncer cerviz, próstata, vesical, colon y linfoma), su uso está descrito en procesos no malignos como en la uropatía asociada a la radioterapia externa, isquemia-estenosis ureteral en trasplante renal $^{1,2,4,5}$, aneurisma de aorta compresivo asî como en pacientes de alto riesgo quirúrgico en los cuales otros procedimientos serían arriesgados o habría resultado fallidos 6,7 .

\section{CASO CLÍNICO}

Varón de 65 años, en hemodiálisis por insuficiencia renal terminal secundaria a poliquistosis hepatorenal. Como otros antecedentes personales de interés, presentaba hipertensión arterial, accidentes cerebrovasculares de repetición con leves secuelas motoras.

Candidato a trasplante renal, en octubre de 2001 se realizó implantación de injerto ortotópico procedente de donante cadáver con anastomosis término-lateral vascular a iliacas y reimplantación ureterovesical sin catéter tutor. El post-operatorio cursó sin incidencias siendo dado de alta con creatinina sérica en torno a $2 \mathrm{mg} / \mathrm{dl}$.

Clínicamente asintomático, en una revisión a los dos meses se advirtió un deterioro de la función renal con ureterohidronefrosis del injerto en la ecografía. Asímismo, se visualizó una colección adyacente al hilio renal de aproximadamente $57 \mathrm{~mm}$.

Tras colocación de una nefrostomía percutánea bajo control ecográfico y drenaje de la colección, se realizó una pielografía anterógrada. En ella se aprecia una estenosis ureteral distal con fístula urinaria asociada, con mínimo paso de contraste a vejiga (Fig. 1).

Tras intento infructuoso de resolución endoscópica, al no lograr el paso de una guía de forma anterógrada o retrógrada a través de la estenosis, optamos por cirugía abierta. Durante la intervención se evidenció una obstrucción completa ureteral severa por amplia fibrosis peri-injerto. Se resecó el segmento estenótico de 1,5 cm de longitud con ureteroneocistotomía tutorizada con catéter DJ. Tras dos semanas se retiró el catéter tutor pero se produjo una re-estenosis del uréter del injerto.

Se ofreció al paciente una reintervención mediante ureteroureterostomía y/o ureteropielostomía, pero ambas posibilidades son rechazadas por el paciente, al presentar alto riesgo quirúrgico dada la comorbilidad asociada.

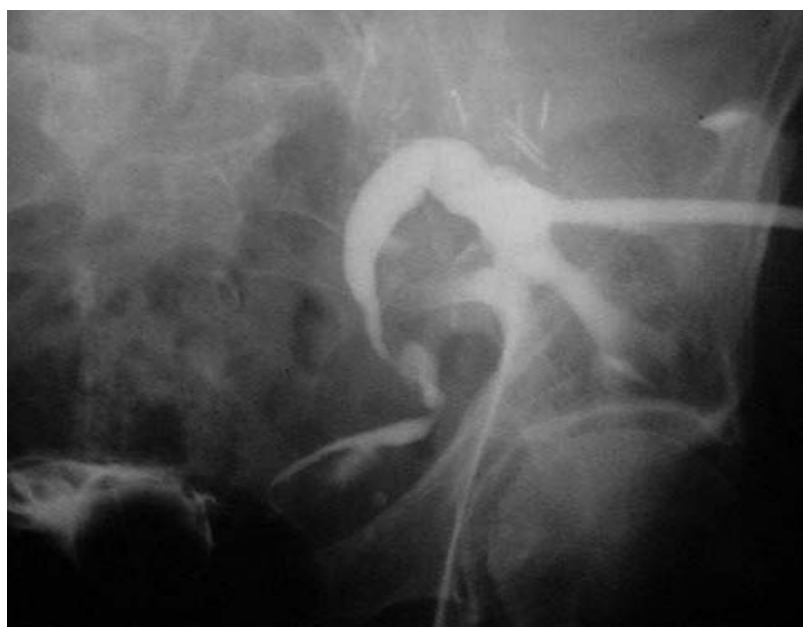

FIGURA 1. Pielografia anterógrada. Estenosis ureteral distal con fistula urinaria asociada. 


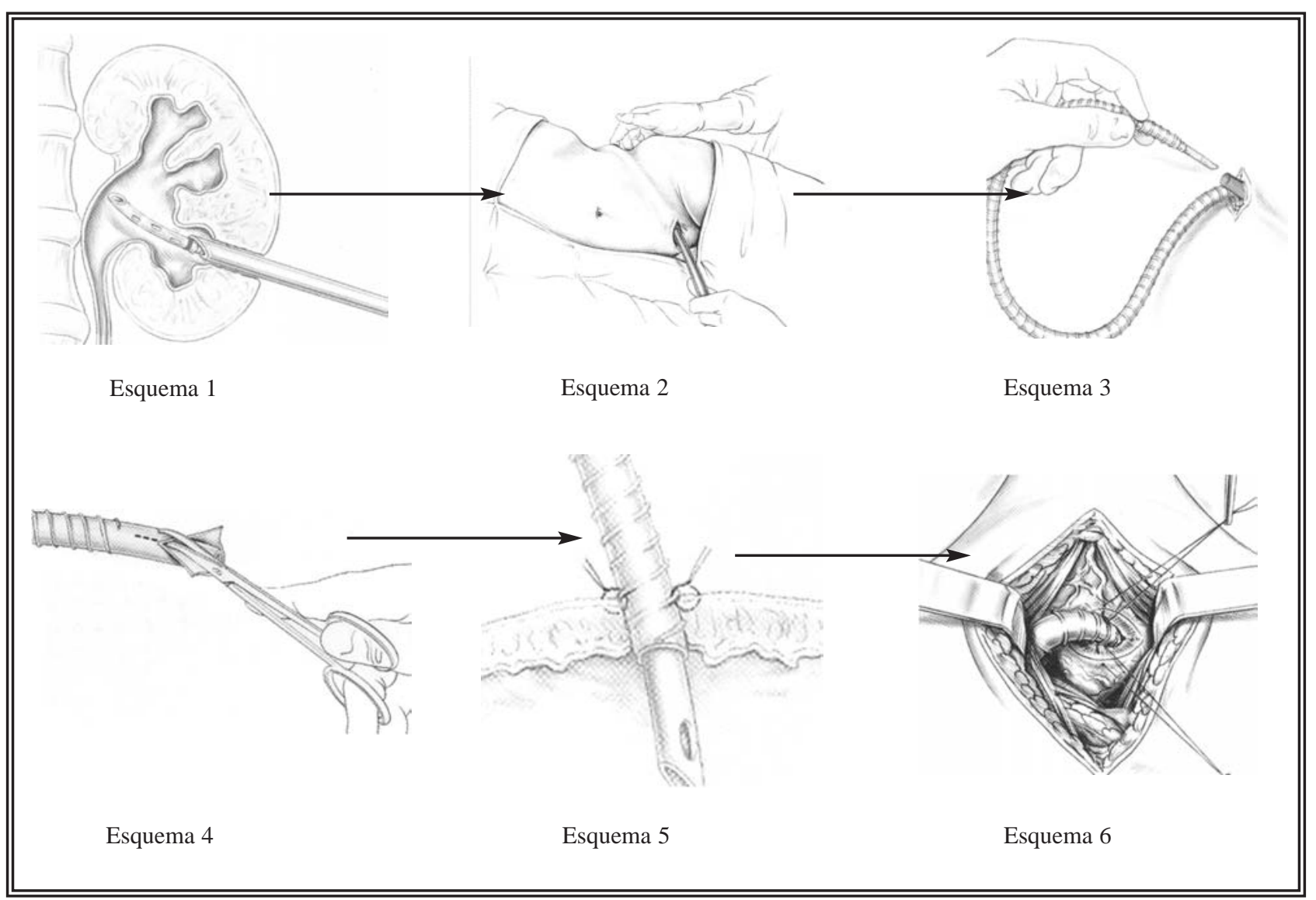

Esquemas 1-6.

Ante la expectativa de portar una nefrostomía permanente, mal tolerada por el paciente, se decidió emplear una derivación urinaria extraanatómica mediante catéter subcutáneo: (Esquemas 1-6).

Bajo anestesia raquídea, se aprovechó el trayecto de la nefrostomía para, bajo control radioscópico, realizar dilataciones sucesivas que permitiesen el paso del stent subcutáneo. Tiene una cubierta de poliéster para mayor adherencia a los tejidos, excepto en sus extremos en contacto con la orina (exclusivamente silicona). Ello proporciona una luz interna de $17 \mathrm{ch}$ y externa de $30 \mathrm{ch}$. A través de una vaina de Amplatz, se introdujo el catéter en pelvis renal. Mediante una pequeña incisión en la fosa iliaca derecha, se tunelizó su extremo distal hasta la zona hipogástrica, donde mediante discreta cistotomía, se introdujo y fijó a la vejiga.

El post-operatorio cursó favorablemente, a excepción de un área de celulitis en trayecto del catéter, resuelta de forma conservadora con anti- bioterapia de amplio espectro 7 días. Fue dado de alta con creatinina basal en torno a $1,8 \mathrm{mg} / \mathrm{dl}$, tras cistouretrografía de control: se observó un reflujo a través del by-pass sin fuga de orina asociada (Fig. 2).

En revisión a 3-6-9-14-20 meses, el paciente se presenta asintomático, comprobándose con analítica, urocultivo, radiografía simple y eco periódicas la ausencia de complicaciones hasta el momento.

\section{DISCUSIÓN}

La derivación urinaria mediante by-pass extra-anatómico se empleó clásicamente en paciente en insuficiencia renal terminal neoplási$\mathrm{ca}^{3}$, en los cuales otras medidas más agresivas están desestimadas o la nefrostomía percutánea es mal tolerada. Está descrito en la literatura su empleo en la uropatía obstructiva no neoplásica como aneurisma de aorta con repercusión urinaria y/o trasplante renal con necrosis ureteral extensa $^{1,2,4,5}$. 


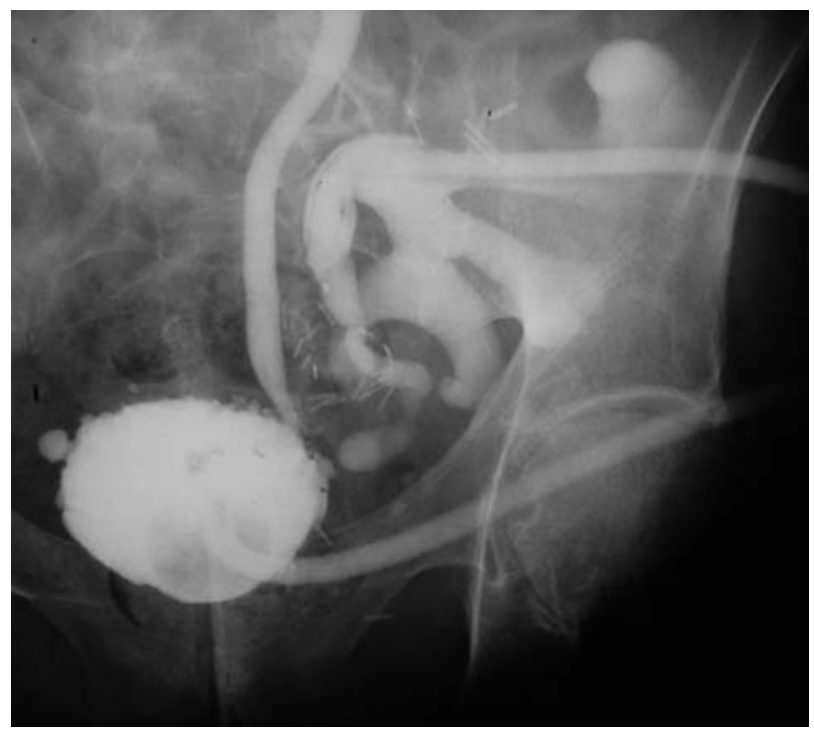

FIGURA 2. CUMS post-operatoria: reflujo a través del catéter subcutáneo sin fuga de orina.

Las complicaciones más habituales de este tipo de catéteres son la obstrucción de su luz por calcificación, infección de la orina y/o movilización ${ }^{8,11}$.

Su uso en trasplantados crea ciertas reservas pues provocan un importante reflujo vesicoureteral en pacientes con cierto grado de inmunodepresión. La profilaxis anbitiótica pre y post-operatoria previene la colonización bacteriana. Mediante el seguimiento periódico con urocultivos, placa de vías urinarias y/o eco, así como control precoz de la infección urinaria, los índices de complicaciones severas son escasos ${ }^{2}$.

Para evitar la obstrucción se emplean catéteres con amplia luz interior. Asociado a una alta ingesta hídrica y control del hiperparatiroidismo secundario del trasplantado, esta posibilidad es muy reducida.

La movilización del catéter está limitada gracias a la cubierta de poliéster que asegura gran adherencia a los tejidos.

Tanto si se produce obstrucción, movilización, infección o rechazo del catéter, puede retirarse o sustituirse a través de una pequeña incisión en la piel ${ }^{8}$.

\section{CONCLUSIONES}

La derivación urinaria mediante catéter subcutáneo es un método sencillo y bien tolerado.
Aunque su indicación fundamental sería paliativa en la insuficiencia renal terminal neoplásica, podría asímismo aplicarse con buenos resultados en pacientes no tumorales (uropatía asociada a la radioterapia externa, isquemiaestenosis ureteral en trasplante renal, aneurisma de aorta compresivo), con alto riesgo quirúrgico o tras el fallo de otras medidas agresivas.

La incidencia de complicaciones es baja y de fácil resolución en su mayoría, de forma conservadora.

\section{REFERENCIAS}

1. DESGRANDCHAMPZ F et al.: Percutaneous extraanatomic nephrovesical diversion: preliminary report. J Endourol 1993; 7: 323-326.

2. DESGRANDCHAMPZ F, PAULHAC P et al.: Artificial uretheral replacement for uretheral necrosis after renal transplantation: report of 3 cases. $J$ Urol 1998; 159: 1830-1832.

3. AHMADZADEH M.: Clinical experience with subcutaneous urinary diversion: new approach using a double pigtail stent. BJU 1991; 67: 596599.

4. NISSENKORN I, YEHOSHUA G.: Nephrovesical subcutaneous stent: an alternative to permanent nephrostomy. J Urol 2000; 163: 528-529.

5. NISSENKORN I, YEHOSHUA G.: RE: nephrovesical subcutaneous stent an alternative to permanent nephrostomy. J Urol 2001; 165: 914.

6. COCKBURN JF et al.: Radiologic insertion of subcutaneous nephrovesical stent for inoperable ureteral obstruction. Am J Roentgenol 1997; 169: 1588-1590.

7. LINGAM K et al.: Subcutaneous urinary diversion, an alternative to percutaneous nephrostomy. J Urol 1994; 152: 72-2.

8. COCKBURN JF et al.: Percutaneous radiologic replacement of blockled nephrovesical stent. Am J Roentgenol 1998; 170: 1109-1110.

9. NAKADA $\mathrm{S}$ et al.: Subcutaneous urinary diversion utilizing a nephrovesical stent, a superior alternative to long-term external drainage? Urology 1995; 45: $538-541$.

10. DI LELIO A.: 2 circumvallate nephro-cystotomy. Arch Ital Urol Nefrol Androl 1992; 64: 45-49.

11. MINHAS S, IRVING HC et al.: Extra-anatomic stents in ureteric obstruction: experience and complications. BJU International 1999; 84: 762764.

Dra. P. Pérez Sanz

C/ Ramón Pérez de Ayala, 92 - 10ํㅡ

28038 Madrid

(Trabajo recibido el 2 septiembre 2003) 